\title{
Crossed Andreev reflection at spin-active interfaces
}

\author{
Mikhail S. Kalenkov \\ I.E. Tamm Department of Theoretical Physics, P.N. Lebedev Physics Institute, 119991 Moscow, Russia \\ Andrei D. Zaikin \\ Forschungszentrum Karlsruhe, Institut für Nanotechnologie, 76021, Karlsruhe, Germany and \\ I.E. Tamm Department of Theoretical Physics, P.N. Lebedev Physics Institute, 119991 Moscow, Russia
}

\begin{abstract}
With the aid of the quasiclassical Eilenberger formalism we develop a theory of non-local electron transport across three-terminal ballistic normal-superconducting-normal (NSN) devices with spinactive NS interfaces. The phenomenon of crossed Andreev reflection (CAR) is known to play the key role in such transport. We demonstrate that CAR is highly sensitive to electron spins and yields a rich variety of properties of non-local conductance which we describe non-perturbatively at arbitrary voltages, temperature, spin-dependent interface transmissions and their polarizations. Our results can be applied to multi-terminal hybrid structures with normal, ferromagnetic and half-metallic electrodes and can be directly tested in future experiments.
\end{abstract}

\section{INTRODUCTION}

Low energy electron transport in hybrid structures composed of a normal metal $(\mathrm{N})$ and a superconductor $(\mathrm{S})$ is governed by Andreev reflection 1 (AR) which causes non-zero subgap conductance ${ }^{2}$ of such structures. AR remains essentially a local effect provided there exists only one NS interface in the system or, else, if the distance between different NS interfaces greatly exceeds the superconducting coherence length $\xi$. If, however, the distance $L$ between two adjacent NS interfaces (i.e. the superconductor size) is smaller than (or comparable with) $\xi$, two additional non-local processes come into play (see Fig. 1). One such process corresponds to direct electron transfer between two N-metals through a superconductor. Another process is the so-called crossed Andreev reflection ${ }^{3.4}$ (CAR): An electron penetrating into the superconductor from the first N-terminal may form a Cooper pair together with another electron from the second $\mathrm{N}$-terminal. In this case a hole will go into the second N-metal and AR becomes a non-local effect. Both these processes contribute to the non-local conductance of hybrid multi-terminal structures which has been directly measured in several recent experiments ${ }^{5,6,7}$.

Theoretically the non-local conductance of NSN hy-

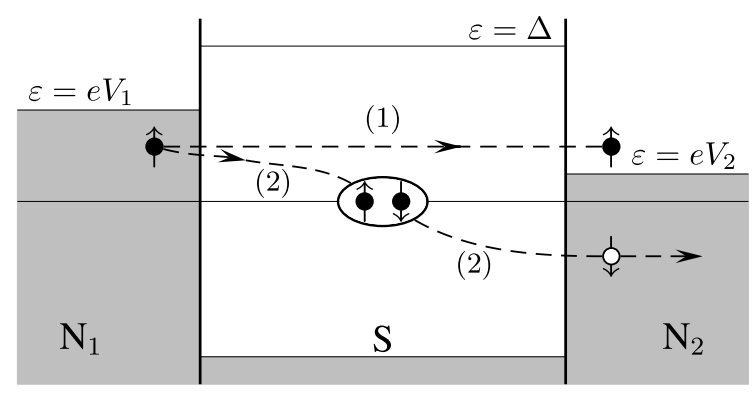

FIG. 1: Two elementary processes contributing to non-local conductance of an NSN device: (1) direct electron transfer and (2) crossed Andreev reflection. brids was analyzed within the perturbation theory in the transmission of NS interfaces in Refs. 8, 9 where it was demonstrated that in the lowest order in the interface transmission and at $T=0 \mathrm{CAR}$ contribution to cross-terminal conductance is exactly canceled by that from elastic electron cotunneling (EC), i.e. the non-local conductance vanishes in this limit. Thus, in order to determine the scale of the effect it is necessary to include higher order (in the barrier transmission) terms into consideration. The corresponding analysis was employed in Refs. 10,11 by means of effective "dressing" of both EC and CAR contributions by higher order processes and more recently by the present authors ${ }^{12}$ with the framework of quasiclassical formalism of Eilenberger equations. We note that the results $\frac{10}{\underline{\underline{10}}}$ and ${ }^{12}$ disagree beyond perturbation theory since "dressing" procedure 10 does not account for all higher order processes. Hence, the approach $\frac{10}{1}$ is in general insufficient to correctly describe non-trivial interplay between normal reflection, tunneling, local AR and CAR to all orders in the interface transmissions. We will return to this issue further below.

Another interesting issue is the effect of disorder. It is well known that disorder enhances interference effects and, hence, can strongly modify local subgap conductance of NS interfaces in the low energy limit13,14,15. Non-local conductance of multi-terminal hybrid NSN structures in the presence of disorder was recently studied in Refs. 16, 17, 18, 19. Brinkman and Golubov made use of the quasiclassical formalism of Usadel equations and proceeded perturbatively in the interface transmissions. Duhot and Melin 18 discussed the impact of weaklocalization-type of effects inside the superconductor on non-local electron transport in NSN structures. Morten et al $\stackrel{17}{\underline{1}}$ employed the circuit theory (thereby going beyond perturbation theory in tunneling) and considered a device with normal terminals attached to a superconductor via an additional normal island (dot) ${ }^{20}$. Very recently a similar structure with a superconducting dot was analyzed ${ }^{19}$ providing a rather general theoretical 
framework to study non-local electron transport in multiterminal NSN structures in the presence of disorder and non-equilibrium effects.

Yet another interesting subject is an interplay between CAR and Coulomb interaction. The effect of electronelectron interactions on AR was investigated in a number of papers $15,21,22$. Interactions should also affect CAR, e.g., by lifting the exact cancellation of EC and CAR contributions ${ }^{23}$ already in the lowest order in tunneling. A complete theory of non-local transport in realistic NSN systems should include both disorder and interactions which remains an important task for future investigations.

An important property of both AR and CAR is that these processes should be sensitive to magnetic properties of normal electrodes because these processes essentially depend on spins of scattered electrons. One possible way to demonstrate spin-resolved CAR is to use ferromagnets (F) instead of normal electrodes ferromagnetsuperconductor-ferromagnet (FSF) structures 5,10,24,25. First experiments on such FSF structures ${ }^{-5}$ illustrated this point by demonstrating the dependence of non-local conductance on the polarization of ferromagnetic terminals. Hence, for better understanding of non-local effects in multi-terminal hybrid proximity structures it is desirable to construct a theory of spin-resolved non-local transport. In the lowest order in tunneling this task was accomplished in Ref. 8. For FSF structures higher orders in the interface transmissions were considered in Refs $\underline{10,11}$.

In this paper we are going to generalize our quasiclassical approach $\frac{12}{2}$ and construct a theory of non-local electron transport in ballistic NSN structures with spinactive interfaces to all orders in their transmissions. Our model allows to distinguish spin-dependent contributions to the non-local conductance and to effectively mimic the situation of ferromagnetic and/or half-metallic electrodes.

The structure of the paper is as follows. In Sec. 2 we introduce our model and discuss the quasiclassical formalism supplemented by the boundary conditions for Green-Keldysh functions which account for electron scattering at spin-active interfaces. In Sec. 3 we employ this formalism and develop a theory of non-local spin-resolved electron transport in NSN structures with spin-active interfaces. Our main conclusions are briefly summarized in Sec. 4.

\section{THE MODEL AND FORMALISM}

Let us consider three-terminal NSN structure depicted in Fig. 2. We will assume that all three metallic electrodes are non-magnetic and ballistic, i.e. the electron elastic mean free path in each metal is larger than any other relevant size scale. In order to resolve spindependent effects we will assume that both NS interfaces are spin-active, i.e. we will distinguish "spin-up" and

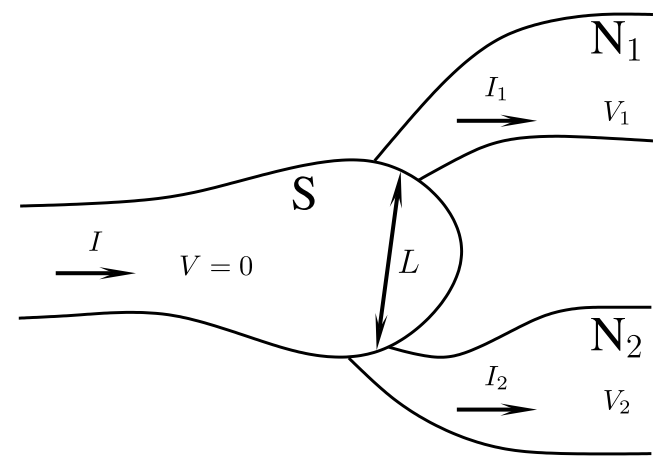

FIG. 2: Schematics of our NSN device.

"spin-down" transmissions of the first $\left(D_{1 \uparrow}\right.$ and $\left.D_{1 \downarrow}\right)$ and the second $\left(D_{2 \uparrow}\right.$ and $\left.D_{2 \downarrow}\right)$ SN interface. All these four transmissions may take any value from zero to one. We also introduce the angle $\varphi$ between polarizations of two interfaces which can take any value between 0 and $2 \pi$.

In what follows effective cross-sections of the two interfaces will be denoted respectively as $\mathcal{A}_{1}$ and $\mathcal{A}_{2}$. The distance between these interfaces $L$ as well as other geometric parameters are assumed to be much larger than $\sqrt{\mathcal{A}_{1,2}}$, i.e. effectively both contacts are metallic constrictions. In this case the voltage drops only across SN interfaces and not inside large metallic electrodes. Hence, nonequilibrium (e.g. charge imbalance) effects related to the electric field penetration into the S-electrode can be neglected. In our analysis we will also disregard Coulomb effects $15,21,22$.

For convenience, we will set the electric potential of the S-electrode equal to zero, $V=0$. In the presence of bias voltages $V_{1}$ and $V_{2}$ applied to two normal electrodes (see Fig. (2) the currents $I_{1}$ and $I_{2}$ will flow through $\mathrm{SN}_{1}$ and $\mathrm{SN}_{2}$ interfaces. These currents can be evaluated with the aid of the quasiclassical formalism of nonequilibrium Green-Eilenberger-Keldysh functions ${ }^{26} \hat{g}^{R, A, K}$ which we briefly specify below.

\section{A. Quasiclassical equations}

In the ballistic limit the corresponding Eilenberger equations take the form

$$
\begin{array}{r}
{\left[\varepsilon \hat{\tau}_{3}+e V(\boldsymbol{r}, t)-\hat{\Delta}(\boldsymbol{r}, t), \hat{g}^{R, A, K}\left(\boldsymbol{p}_{F}, \varepsilon, \boldsymbol{r}, t\right)\right]+} \\
+i \boldsymbol{v}_{F} \nabla \hat{g}^{R, A, K}\left(\boldsymbol{p}_{F}, \varepsilon, \boldsymbol{r}, t\right)=0
\end{array}
$$

where $[\hat{a}, \hat{b}]=\hat{a} \hat{b}-\hat{b} \hat{a}, \varepsilon$ is the quasiparticle energy, $\boldsymbol{p}_{F}=$ $m \boldsymbol{v}_{F}$ is the electron Fermi momentum vector and $\hat{\tau}_{3}$ is the Pauli matrix in Nambu space. The functions $\hat{g}^{R, A, K}$ also obey the normalization conditions $\left(\hat{g}^{R}\right)^{2}=\left(\hat{g}^{A}\right)^{2}=1$ and $\hat{g}^{R} \hat{g}^{K}+\hat{g}^{K} \hat{g}^{A}=0$. Here and below the product of matrices is defined as time convolution.

Green functions $\hat{g}^{R, A, K}$ and $\hat{\Delta}$ are $4 \times 4$ matrices in Nambu and spin spaces. In Nambu space they can be 
parameterized as

$$
\hat{g}^{R, A, K}=\left(\begin{array}{ll}
g^{R, A, K} & f^{R, A, K} \\
\tilde{f}^{R, A, K} & \tilde{g}^{R, A, K}
\end{array}\right), \quad \hat{\Delta}=\left(\begin{array}{cc}
0 & \Delta i \sigma_{2} \\
\Delta^{*} i \sigma_{2} & 0
\end{array}\right),
$$

where $g^{R, A, K}, f^{R, A, K}, \tilde{f}^{R, A, K}, \tilde{g}^{R, A, K}$ are $2 \times 2$ matrices in the spin space, $\Delta$ is the BCS order parameter and $\sigma_{i}$ are Pauli matrices. For simplicity we will only consider the case of spin-singlet isotropic pairing in the superconducting electrode. The current density is related to the Keldysh function $\hat{g}^{K}$ according to the standard relation

$$
\boldsymbol{j}(\boldsymbol{r}, t)=-\frac{e N_{0}}{8} \int d \varepsilon\left\langle\boldsymbol{v}_{F} \operatorname{Sp}\left[\hat{\tau}_{3} \hat{g}^{K}\left(\boldsymbol{p}_{F}, \varepsilon, \boldsymbol{r}, t\right)\right]\right\rangle,
$$

where $N_{0}=m p_{F} / 2 \pi^{2}$ is the density of state at the Fermi level and angular brackets $\langle\ldots\rangle$ denote averaging over the Fermi momentum.

\section{B. Riccati parameterization}

The above matrix Green-Keldysh functions can be conveniently parameterized by four Riccati amplitudes $\gamma^{R, A}$, $\tilde{\gamma}^{R, A}$ and two "distribution functions" $x^{K}, \tilde{x}^{K}$ (here and below we chose to follow the notations ${ }^{30}$ ):

$$
\hat{g}^{K}=2 \hat{N}^{R}\left(\begin{array}{cc}
x^{K}-\gamma^{R} \tilde{x}^{K} \tilde{\gamma}^{A} & -\gamma^{R} \tilde{x}^{K}+x^{K} \gamma^{A} \\
-\tilde{\gamma}^{R} x^{K}+\tilde{x}^{K} \tilde{\gamma}^{A} & \tilde{x}^{K}-\tilde{\gamma}^{R} x^{K} \gamma^{A}
\end{array}\right) \hat{N}^{A},
$$

where functions $\gamma^{R, A}$ and $\tilde{\gamma}^{R, A}$ are Riccati amplitudes

$$
\hat{g}^{R, A}= \pm \hat{N}^{R, A}\left(\begin{array}{cc}
1+\gamma^{R, A} \tilde{\gamma}^{R, A} & 2 \gamma^{R, A} \\
-2 \tilde{\gamma}^{R, A} & -1-\tilde{\gamma}^{R, A} \gamma^{R, A}
\end{array}\right)
$$

and $\hat{N}^{R, A}$ are the following matrices

$$
\hat{N}^{R, A}=\left(\begin{array}{cc}
\left(1-\gamma^{R, A} \tilde{\gamma}^{R, A}\right)^{-1} & 0 \\
0 & \left(1-\tilde{\gamma}^{R, A} \gamma^{R, A}\right)^{-1}
\end{array}\right) .
$$

With the aid of the above parameterization one can identically transform the quasiclassical equations (11) into the following set of effectively decoupled equations for Riccati amplitudes and distribution functions 30

$$
\begin{array}{r}
i \boldsymbol{v}_{F} \nabla \gamma^{R, A}+[\varepsilon+e V(\boldsymbol{r}, t)] \gamma^{R, A}+\gamma^{R, A}[\varepsilon-e V(\boldsymbol{r}, t)] \\
=\gamma^{R, A} \Delta^{*} i \sigma_{2} \gamma^{R, A}-\Delta i \sigma_{2}, \\
i \boldsymbol{v}_{F} \nabla \tilde{\gamma}^{R, A}-[\varepsilon-e V(\boldsymbol{r}, t)] \tilde{\gamma}^{R, A}-\tilde{\gamma}^{R, A}[\varepsilon+e V(\boldsymbol{r}, t)] \\
=\tilde{\gamma}^{R, A} \Delta i \sigma_{2} \tilde{\gamma}^{R, A}-\Delta^{*} i \sigma_{2}, \\
i \boldsymbol{v}_{F} \nabla x^{K}+[\varepsilon+e V(\boldsymbol{r}, t)] x^{K}-x^{K}[\varepsilon+e V(\boldsymbol{r}, t)] \\
-\gamma^{R} \Delta^{*} i \sigma_{2} x^{K}-x^{K} \Delta i \sigma_{2} \tilde{\gamma}^{A}=0, \\
i \boldsymbol{v}_{F} \nabla \tilde{x}^{K}-[\varepsilon-e V(\boldsymbol{r}, t)] \tilde{x}^{K}+\tilde{x}^{K}[\varepsilon-e V(\boldsymbol{r}, t)] \\
-\tilde{\gamma}^{R} \Delta i \sigma_{2} \tilde{x}^{K}-\tilde{x}^{K} \Delta^{*} i \sigma_{2} \gamma^{A}=0 .
\end{array}
$$

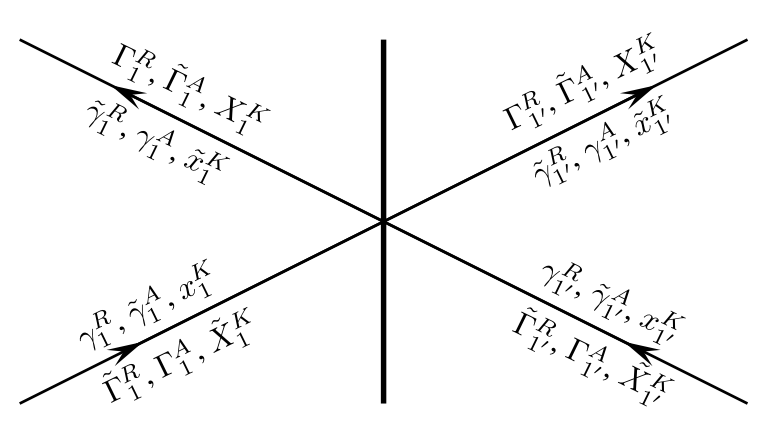

FIG. 3: Riccati amplitudes for incoming and outgoing trajectories from the both sides of the interface.

Depending on the particular trajectory it is also convenient to introduce a "replica" of both Riccati amplitudes and distribution functions which - again following the notations 30.31 - will be denoted by capital letters $\Gamma$ and $X$. These "capital" Riccati amplitudes and distribution functions obey the same equations (7)-(10) with the replacement $\gamma \rightarrow \Gamma$ and $x \rightarrow X$. The distinction between different Riccati amplitudes and distribution functions will be made explicit below.

\section{Boundary conditions}

Quasiclassical equations should be supplemented by appropriate boundary conditions at metallic interfaces. In the case of specularly reflecting spin-degenerate interfaces these conditions were derived by Zaitsev ${ }^{27}$ and later generalized to spin-active interfaces in Ref. 32 .

Before specifying these conditions it is important to emphasize that the applicability of the Eilenberger quasiclassical formalism with appropriate boundary conditions to hybrid structures with two or more barriers is, in general, a non-trivial issue 28,29 . Electrons scattered at different barriers interfere and form bound states (resonances) which cannot be correctly described within the quasiclassical formalism employing Zaitsev boundary conditions or their direct generalization. Here we avoid this problem by choosing the appropriate geometry of our NSN device, see Fig. 2. In our system any relevant trajectory reaches each NS interface only once whereas the probability of multiple reflections at both interfaces is small in the parameter $\mathcal{A}_{1} \mathcal{A}_{2} / L^{4} \ll 1$. Hence, resonances formed by multiply reflected electron waves can be neglected, and our formalism remains adequate for the problem in question.

It will be convenient for us to formulate the boundary conditions directly in terms of Riccati amplitudes and the distribution functions. Let us consider the first NS interface and explicitly specify the relations between Riccati amplitudes and distribution functions for incoming and outgoing trajectories, see Fig. 3 . The boundary con- 
ditions for $\Gamma_{1}^{R}, \tilde{\Gamma}_{1}^{A}$ and $X_{1}^{K}$ can be written in the form ${ }^{31}$

$$
\begin{gathered}
\Gamma_{1}^{R}=r_{1 l}^{R} \gamma_{1}^{R} \underline{S}_{11}^{+}+t_{1 l}^{R} \gamma_{1^{\prime}}^{R} \underline{S}_{11^{\prime}}^{+}, \\
\tilde{\Gamma}_{1}^{A}=\underline{S}_{11} \tilde{\gamma}_{1}^{A} \tilde{r}_{1 r}^{A}+\underline{S}_{11^{\prime}} \tilde{\gamma}_{1^{\prime}}^{A} \tilde{t}_{1 r}^{A}, \\
X_{1}^{K}=r_{1 l}^{R} x_{1}^{K} \tilde{r}_{1 r}^{A}+t_{1 l}^{R} x_{1^{\prime}}^{K} \tilde{t}_{1 r}^{A}-a_{1 l}^{R} \tilde{x}_{1^{\prime}}^{K} \tilde{a}_{1 r}^{A} .
\end{gathered}
$$

Here we defined the transmission $(t)$, reflection $(r)$, and branch-conversion $(a)$ amplitudes as:

$$
\begin{gathered}
r_{1 l}^{R}=+\left[\left(\beta_{1^{\prime} 1}^{R}\right)^{-1} S_{11}^{+}-\left(\beta_{1^{\prime} 1^{\prime}}^{R}\right)^{-1} S_{1^{\prime}}^{+}\right]^{-1}\left(\beta_{1^{\prime} 1}^{R}\right)^{-1} \\
t_{1 l}^{R}=-\left[\left(\beta_{1^{\prime} 1}^{R}\right)^{-1} S_{11}^{+}-\left(\beta_{1^{\prime} 1^{\prime}}^{R}\right)^{-1} S_{11^{\prime}}^{+}\right]^{-1}\left(\beta_{1^{\prime} 1^{\prime}}^{R}\right)^{-1} \\
\tilde{r}_{1 r}^{A}=+\left(\beta_{1^{\prime} 1}^{A}\right)^{-1}\left[S_{11}\left(\beta_{1^{\prime} 1}^{A}\right)^{-1}-S_{11^{\prime}}\left(\beta_{1^{\prime} 1^{\prime}}^{A}\right)^{-1}\right]^{-1} \\
\tilde{t}_{1 r}^{A}=-\left(\beta_{1^{\prime} 1^{\prime}}^{A}\right)^{-1}\left[S_{11}\left(\beta_{1^{\prime} 1}^{A}\right)^{-1}-S_{11^{\prime}}\left(\beta_{1^{\prime} 1^{\prime}}^{A}\right)^{-1}\right]^{-1} \\
a_{1 l}^{R}=\left(\Gamma_{1}^{R} \underline{S}_{11}-S_{11} \gamma_{1}^{R}\right)\left(\tilde{\beta}_{11^{\prime}}^{R}\right)^{-1} \\
\tilde{a}_{1 r}^{A}=\left(\tilde{\beta}_{11^{\prime}}^{A}\right)^{-1}\left(\underline{S}_{11}^{+} \tilde{\Gamma}_{1}^{A}-\tilde{\gamma}_{1}^{A} S_{11}^{+}\right)
\end{gathered}
$$

where

$$
\begin{aligned}
& \beta_{i j}^{R}=S_{i j}^{+}-\gamma_{j}^{R} \underline{S}_{i j}^{+} \tilde{\gamma}_{i}^{R}, \tilde{\beta}_{i j}^{R}=\underline{S}_{j i}-\tilde{\gamma}_{j}^{R} S_{j i} \gamma_{i}^{R}, \\
& \beta_{i j}^{A}=S_{i j}-\gamma_{i}^{A} \underline{S}_{i j} \tilde{\gamma}_{j}^{A}, \tilde{\beta}_{i j}^{A}=\underline{S}_{j i}^{+}-\tilde{\gamma}_{i}^{A} S_{j i}^{+} \gamma_{j}^{A} .
\end{aligned}
$$

Similarly, the boundary conditions for $\tilde{\Gamma}_{1}^{R}, \Gamma_{1}^{A}$, and $\tilde{X}_{1}^{K}$ take the form:

$$
\begin{gathered}
\tilde{\Gamma}_{1}^{R}=\tilde{r}_{1 l}^{R} \tilde{\gamma}_{1}^{R} S_{11}+\tilde{t}_{1 l}^{R} \tilde{\gamma}_{1^{\prime}}^{R} S_{1^{\prime} 1}, \\
\Gamma_{1}^{A}=S_{11}^{+} \gamma_{1}^{A} r_{1 r}^{A}+S_{1^{\prime} 1}^{+} \gamma_{1^{\prime}}^{A} t_{1 r}^{A}, \\
\tilde{X}_{1}^{K}=\tilde{r}_{1 l}^{R} \tilde{x}_{1}^{K} r_{1 r}^{A}+\tilde{t}_{1 l}^{R} \tilde{x}_{1^{\prime}}^{K} t_{1 r}^{A}-\tilde{a}_{1 l}^{R} x_{1^{\prime}}^{K} a_{1 r}^{A},
\end{gathered}
$$

where

$$
\begin{gathered}
\tilde{r}_{1 l}^{R}=+\left[\left(\tilde{\beta}_{1^{\prime} 1}^{R}\right)^{-1} \underline{S}_{11}-\left(\tilde{\beta}_{1^{\prime} 1^{\prime}}^{R}\right)^{-1} \underline{S}_{1^{\prime} 1}\right]^{-1}\left(\tilde{\beta}_{1^{\prime} 1}^{R}\right)^{-1}, \\
t_{1 l}^{R}=-\left[\left(\tilde{\beta}_{1^{\prime} 1}^{R}\right)^{-1} \underline{S}_{11}-\left(\tilde{\beta}_{1^{\prime} 1^{\prime}}^{R}\right)^{-1} \underline{S}_{1^{\prime} 1}\right]^{-1}\left(\tilde{\beta}_{1^{\prime} 1^{\prime}}^{R}\right)^{-1}, \\
r_{1 r}^{A}=+\left(\tilde{\beta}_{1^{\prime} 1}^{A}\right)^{-1}\left[\underline{S}_{11}^{+}\left(\tilde{\beta}_{1^{\prime} 1}^{A}\right)^{-1}-\underline{S}_{1^{\prime} 1}^{+}\left(\tilde{\beta}_{1^{\prime} 1^{\prime}{ }^{\prime}}^{A}\right)^{-1}\right]^{-1}, \\
\tilde{t}_{1 r}^{A}=-\left(\tilde{\beta}_{1^{\prime} 1^{\prime}}^{A}\right)^{-1}\left[\underline{S}_{11}^{+}\left(\tilde{\beta}_{1^{\prime} 1}^{A}\right)^{-1}-\underline{S}_{1^{\prime} 1}^{+}\left(\tilde{\beta}_{1^{\prime} 1^{\prime}}^{A}\right)^{-1}\right]^{-1}, \\
\tilde{a}_{1 l}^{R}=\left(\tilde{\Gamma}_{1}^{R} S_{11}^{+}-\underline{S}_{11}^{+} \tilde{\gamma}_{1}^{R}\right)\left(\beta_{11^{\prime}}^{R}\right)^{-1} \\
a_{1 r}^{A}=\left(\beta_{11^{\prime}}^{A}\right)^{-1}\left(S_{11} \Gamma_{1}^{A}-\gamma_{1}^{A} \underline{S}_{11}\right) .
\end{gathered}
$$

Boundary conditions for $\Gamma_{1^{\prime}}^{R, A}, \tilde{\Gamma}_{1^{\prime}}^{R, A}, X_{1^{\prime}}^{K}$ and $\tilde{X}_{1^{\prime}}^{K}$ can be obtained from the above equations simply by replacing $1 \leftrightarrow 1^{\prime}$.

The matrices $S_{11}, S_{11^{\prime}}, S_{1^{\prime} 1}$, and $S_{1^{\prime} 1^{\prime}}$ constitute the components of the $\mathcal{S}$-matrix describing electron scattering at the first interface:

$$
\mathcal{S}=\left(\begin{array}{cc}
S_{11} & S_{11^{\prime}} \\
S_{1^{\prime} 1} & S_{1^{\prime} 1^{\prime}}
\end{array}\right), \quad \mathcal{S S}^{+}=1
$$

In our three terminal geometry nonlocal conductance arises only from trajectories that cross both interfaces, as illustrated in Fig. 4. Accordingly, the above boundary conditions should be employed at both NS interfaces.

Finally, one needs to specify the asymptotic boundary conditions far from NS interfaces. Deep in metallic electrodes we have

$$
\begin{gathered}
\gamma_{1}^{R}=\tilde{\gamma}_{1}^{R}=\gamma_{1}^{A}=\tilde{\gamma}_{1}^{A}=0, \\
x_{1}^{K}=h_{0}\left(\varepsilon+e V_{1}\right), \quad \tilde{x}_{1}^{K}=-h_{0}\left(\varepsilon-e V_{1}\right), \\
\gamma_{2}^{R}=\tilde{\gamma}_{2}^{R}=\gamma_{2}^{A}=\tilde{\gamma}_{2}^{A}=0, \\
x_{2}^{K}=h_{0}\left(\varepsilon+e V_{2}\right), \quad \tilde{x}_{2}^{K}=-h_{0}\left(\varepsilon-e V_{2}\right),
\end{gathered}
$$

where $h_{0}(\varepsilon)=\tanh (\varepsilon / 2 T)$ - equilibrium distribution function. In the bulk of superconducting electrode we have

$$
\begin{gathered}
\tilde{\gamma}_{1^{\prime}}^{R}=-a(\varepsilon) i \sigma_{2}, \quad \gamma_{1^{\prime}}^{A}=a^{*}(\varepsilon) i \sigma_{2}, \\
\tilde{x}_{1^{\prime}}^{K}=-\left[1-|a(\varepsilon)|^{2}\right] h_{0}(\varepsilon), \\
\gamma_{2^{\prime}}^{R}=a(\varepsilon) i \sigma_{2}, \quad \tilde{\gamma}_{2^{\prime}}^{A}=-a^{*}(\varepsilon) i \sigma_{2}, \\
x_{2^{\prime}}^{K}=\left[1-|a(\varepsilon)|^{2}\right] h_{0}(\varepsilon),
\end{gathered}
$$

where we denoted $a(\varepsilon)=-\left(\varepsilon-\sqrt{\varepsilon^{2}-\Delta^{2}}\right) / \Delta$.

\section{Green functions}

With the aid of the above equations and boundary conditions it is straightforward to evaluate the quasiclassical Green-Keldysh functions for our three-terminal device along any trajectory of interest. For instance, from the boundary conditions at the second interface we find

$$
\Gamma_{2^{\prime}}^{R}=i a(\varepsilon) A_{2} \sigma_{2},
$$

where $A_{2}=S_{2^{\prime} 2^{\prime}} \sigma_{2} \underline{S}_{2^{\prime} 2^{\prime}}^{+} \sigma_{2}$. Integrating Eq. (7) along the trajectory connecting both interfaces and using Eq. (40) as the initial condition we immediately evaluate the Riccati amplitude at the first interface:

$$
\begin{gathered}
\gamma_{1^{\prime}}^{R}=i \frac{a A_{2}+\left(a A_{2} \varepsilon+\Delta\right) Q}{1-\left(a A_{2} \Delta+\varepsilon\right) Q} \sigma_{2}, \\
Q=\frac{\tanh \left[i \Omega L / v_{F}\right]}{\Omega}, \quad \Omega=\sqrt{\varepsilon^{2}-\Delta^{2}} .
\end{gathered}
$$

Employing the boundary conditions again we obtain

$$
\begin{gathered}
\Gamma_{1}^{R}=i S_{11^{\prime}} K_{21}^{-1}\left[a A_{2}+\left(a A_{2} \varepsilon+\Delta\right) Q\right] \sigma_{2} \underline{S}_{11^{\prime}}^{+}, \\
\tilde{\Gamma}_{1}^{R}=-i a \underline{S}_{1^{\prime} 1}^{+} \sigma_{2} S_{1^{\prime} 1^{\prime}} K_{21}^{-1}\left[1-\left(a A_{2} \Delta+\varepsilon\right) Q\right] S_{1^{\prime} 1^{\prime}}^{-1} S_{1^{\prime} 1},
\end{gathered}
$$

where

$$
\begin{gathered}
K_{i j}=\left(1-a^{2} A_{i} A_{j}\right)-\left[\varepsilon\left(1+a^{2} A_{i} A_{j}\right)+\Delta a\left(A_{i}+A_{j}\right)\right] Q, \\
A_{1}=\sigma_{2} \underline{S}_{1^{\prime} 1^{\prime}}^{+} \sigma_{2} S_{1^{\prime} 1^{\prime}} .
\end{gathered}
$$

We also note that the relation $\left(\Gamma^{R, A}\right)^{+}=\tilde{\Gamma}^{A, R}$ and $\left(\gamma^{R, A}\right)^{+}=\tilde{\gamma}^{A, R}$ makes it unnecessary (while redundant) to separately calculate the advanced Riccati amplitudes. 


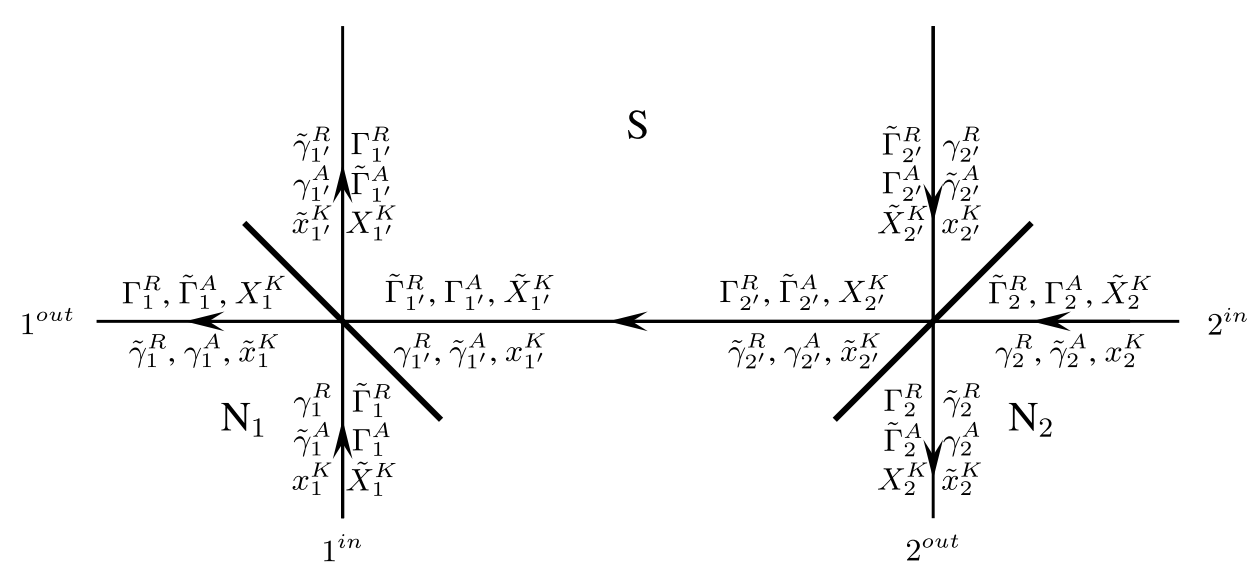

FIG. 4: Riccati amplitudes for incoming and outgoing trajectories for an NSN structure with two barriers. The arrows define quasiparticle momentum directions. We also indicate relevant Riccati amplitudes and distribution functions parameterizing the Green-Keldysh function for the corresponding trajectory.

Let us now evaluate the distribution functions at both interfaces. With the aid of the boundary conditions at the second interface we obtain

$$
\begin{aligned}
X_{2^{\prime}}^{K}=S_{2^{\prime} 2^{\prime}} S_{2^{\prime} 2^{\prime}}^{+} & \left(1-|a|^{2}\right) h_{0}(\varepsilon)+S_{2^{\prime} 2} S_{2^{\prime} 2}^{+} x_{2}^{K}- \\
& -|a|^{2} S_{2^{\prime} 2^{\prime}} \sigma_{2} \underline{S}_{22^{\prime}}^{+} \underline{S}_{22^{\prime}} \sigma_{2} S_{2^{\prime} 2^{\prime}}^{+} \tilde{x}_{2}^{K} .
\end{aligned}
$$

Integrating Eq. (9) along the trajectory connecting both interfaces with initial condition for $X_{2^{\prime}}^{K}$, we arrive at the expression for $x_{1^{\prime}}^{K}$

$$
\begin{aligned}
& x_{1^{\prime}}^{K}=\left[1-\left(a A_{2} \Delta+\varepsilon\right) Q\right]^{-1} X_{2^{\prime}}^{K} \times \\
& \quad \times\left(1-\tanh ^{2} i L \Omega / v_{F}\right)\left[1-\left(a A_{2} \Delta+\varepsilon\right) Q\right]^{+^{-1}} .
\end{aligned}
$$

Then we can find distribution functions at the first interface. On the normal metal side of the interface we find

$$
X_{1}^{K}=r_{1 l}^{R} x_{1}^{K} r_{1 l}^{R^{+}}+t_{1 l}^{R} x_{1^{\prime}}^{K} t_{1 l}^{R^{+}}+a_{1 l}^{R} a_{1 l}^{R^{+}}\left(1-|a|^{2}\right) h_{0}(\varepsilon)
$$

where

$$
\begin{aligned}
& r_{1 l}^{R}= S_{11^{\prime}} K_{21}^{-1}\left[\left(1-\left(a A_{2} \Delta+\varepsilon\right) Q\right) S_{1^{\prime} 1^{\prime}}^{+} S_{1^{\prime} 1}^{+-1}-\right. \\
&\left.-a\left(a A_{2}+\left(a A_{2} \varepsilon+\Delta\right) Q\right) \sigma_{2} \underline{S}_{1^{\prime} 1^{\prime}}^{+} \sigma_{2} S_{1^{\prime} 1}^{+-1}\right], \\
& t_{1 l}^{R}=S_{11^{\prime}} K_{21}^{-1}\left(1-\left(a A_{2} \Delta+\varepsilon\right) Q\right), \\
& a_{1 l}^{R}=i S_{11^{\prime}} K_{21}^{-1}\left(a A_{2}+\left(a A_{2} \varepsilon+\Delta\right) Q\right) \sigma_{2} \underline{S}_{1^{\prime} 1^{\prime}}^{+}
\end{aligned}
$$

The corresponding expression for $\tilde{X}_{1}^{K}$ is obtained analogously. We get

$$
\tilde{X}_{1}^{K}=\tilde{r}_{1 l}^{R} \tilde{x}_{1}^{K} \tilde{r}_{1 l}^{R^{+}}-\tilde{t}_{1 l}^{R} \tilde{t}_{1 l}^{R^{+}}\left(1-|a|^{2}\right) h_{0}(\varepsilon)-\tilde{a}_{1 l}^{R} x_{1^{\prime}}^{K} \tilde{a}_{1 l}^{R^{+}} .
$$

where

$$
\begin{gathered}
\tilde{r}_{1 l}^{R}=-\left[\underline{S}_{1^{\prime} 1}^{-1} \underline{S}_{1^{\prime} 1^{\prime}} \sigma_{2}\left(1-\left(a A_{2} \Delta+\varepsilon\right) Q\right)-\right. \\
\left.-\underline{S}_{1^{\prime} 1}^{-1} \sigma_{2} S_{1^{\prime} 1^{\prime}} a\left(a A_{2}+\left(a A_{2} \varepsilon+\Delta\right) Q\right)\right] K_{12}^{-1} \sigma_{2} \underline{S}_{11^{\prime}}^{+}, \\
\tilde{t}_{1 l}^{R}=\underline{S}_{1^{\prime} 1}^{+} \underline{S}_{1^{\prime} 1^{\prime}}^{+1} \sigma_{2}\left(1-\left(a A_{2} \Delta+\varepsilon\right) Q\right) K_{12}^{-1} \sigma_{2} \underline{S}_{1^{\prime} 1^{\prime}}^{+}, \\
\tilde{a}_{1 l}^{R}=i a \underline{S}_{1^{\prime} 1}^{+} \sigma_{2} S_{1^{\prime} 1^{\prime}} K_{21}^{-1}\left(1-\left(a A_{2} \Delta+\varepsilon\right) Q\right) .
\end{gathered}
$$

Combining the above results for the Riccati amplitudes and the distribution functions we can easily evaluate the Keldysh Green function at the first interface. For instance, for the trajectory $1^{\text {out }}$ (see Fig. (4) we obtain

$$
g_{1 \text { out }}^{K}=2\left(X_{1}^{K}-\Gamma_{1}^{R} \tilde{x}_{1}^{K} \Gamma_{1}^{R^{+}}\right), \quad \tilde{g}_{1^{\text {out }}}^{K}=2 \tilde{x}_{1}^{K} .
$$

The Keldysh Green function for the trajectory $1^{\text {in }}$ is evaluated analogously, and we get

$$
g_{1 \text { in }}^{K}=2 x_{1}^{K}, \quad \tilde{g}_{1^{\text {in }}}^{K}=2\left(\tilde{X}_{1}^{K}-\tilde{\Gamma}_{1}^{R} x_{1}^{K} \tilde{\Gamma}_{1}^{R^{+}}\right) .
$$

\section{NONLOCAL CONDUCTANCE}

\section{A. General results}

Now we are ready to evaluate the current $I_{1}$ across the first interface. This current takes the form:

$$
I_{1}=I_{1}^{B T K}\left(V_{1}\right)-\frac{G_{0}}{8 e} \int d \varepsilon \operatorname{Sp}\left(\hat{\tau}_{3} \hat{g}_{1^{\text {out }}}^{K}-\hat{\tau}_{3} \hat{g}_{1^{\text {in }}}^{K}\right),
$$

where

$$
G_{0}=\frac{8 \gamma_{1} \gamma_{2} \mathcal{N}_{1} \mathcal{N}_{2}}{R_{q} p_{F}^{2} L^{2}}
$$

is the normal state nonlocal conductance of our device at fully transparent interfaces, $p_{F} \gamma_{1(2)}$ is normal to the 
first (second) interface component of the Fermi momentum for electrons propagating straight between the interfaces, $\mathcal{N}_{1,2}=p_{F}^{2} \mathcal{A}_{1,2} / 4 \pi$ define the number of conducting channels of the corresponding interface, $R_{q}=2 \pi / e^{2}$ is the quantum resistance unit.

Here $I_{1}^{B T K}\left(V_{1}\right)$ stands for the contribution to the current through the first interface coming from trajectories that never cross the second interface. This is just the standard BTK contribution ${ }^{2,31}$. The non-trivial contribution is represented by the last term in Eq. (59) which accounts for the presence of the second NS interface. We observe that this non-local contribution to the current is small as $\propto 1 / p_{F}^{2} L^{2}$ (rather than $\propto 1 / p_{F}^{3} L^{3}$ as suggested in Ref. 24). This term will be analyzed in details below.

The functions $\hat{g}_{1 \text { in }}^{K}$ and $\hat{g}_{1 \text { out }}^{K}$ are the Keldysh Green functions evaluated on the trajectories $1^{\text {in }}$ and $1^{\text {out }}$ respectively. Using the above expression for the Riccati amplitudes and the distribution functions we find

$$
\begin{gathered}
\operatorname{Sp}\left(\hat{\tau}_{3} \hat{g}_{1^{\text {out }}}^{K}-\hat{\tau}_{3} \hat{g}_{1^{\text {in }}}^{K}\right)= \\
=2 \operatorname{Sp}\left[r_{1 l}^{R} r_{1 l}^{R^{+}}-\tilde{\Gamma}_{1}^{R} \tilde{\Gamma}_{1}^{R^{+}}-1\right]\left(h_{0}\left(\varepsilon+e V_{1}\right)-h_{0}(\varepsilon)\right)- \\
-2 \operatorname{Sp}\left[\tilde{r}_{1 l}^{R} \tilde{r}_{1 l}^{R^{+}}-\Gamma_{1}^{R} \Gamma_{1}^{R^{+}}-1\right]\left(h_{0}\left(\varepsilon-e V_{1}\right)-h_{0}(\varepsilon)\right)+ \\
\quad+2\left(1-\tanh ^{2} i L \Omega / v_{F}\right) \times \\
\times \operatorname{Sp}\left[K _ { 2 1 } ^ { - 1 } \left\{S_{2^{\prime} 2} S_{2^{\prime} 2}^{+}\left(h_{0}\left(\varepsilon+e V_{2}\right)-h_{0}(\varepsilon)\right)+\right.\right. \\
\left.+|a|^{2} S_{2^{\prime} 2^{\prime}} \sigma_{2} \underline{S}_{22^{\prime}}^{+} \underline{S}_{22^{\prime}} \sigma_{2} S_{2^{\prime} 2^{\prime}}^{+}\left(h_{0}\left(\varepsilon-e V_{2}\right)-h_{0}(\varepsilon)\right)\right\} K_{21}^{+1} \times \\
\left.\quad \times\left(S_{11^{\prime}}^{+} S_{11^{\prime}}-\left|a^{2}\right| S_{1^{\prime} 1^{\prime}}^{+} \sigma_{2} \underline{S}_{1^{\prime} 1} \underline{S}_{1^{\prime} 1}^{+} \sigma_{2} S_{1^{\prime} 1^{\prime}}\right)\right], \quad(61)
\end{gathered}
$$

where we explicitly used the fact that in equilibrium $\operatorname{Sp}\left(\hat{\tau}_{3} \hat{g}_{1 \text { out }}^{K}-\hat{\tau}_{3} \hat{g}_{1^{\text {in }}}^{K}\right) \equiv 0$. Substituting (61) into (59), we finally obtain

$$
I_{1}=I_{1}^{B T K}\left(V_{1}\right)+I_{11}\left(V_{1}\right)+I_{12}\left(V_{2}\right) .
$$

The correction to the local BTK current (arising from trajectories crossing also the second NS interface) has the following form

$$
\begin{aligned}
& I_{11}\left(V_{1}\right)=-\frac{G_{0}}{4 e} \int d \varepsilon\{ \\
& \operatorname{Sp}\left[r_{1 l}^{R} r_{1 l}^{R^{+}}-\tilde{\Gamma}_{1}^{R} \tilde{\Gamma}_{1}^{R^{+}}-1\right]\left(h_{0}\left(\varepsilon+e V_{1}\right)-h_{0}(\varepsilon)\right)- \\
& \left.-\operatorname{Sp}\left[\tilde{r}_{1 l}^{R} \tilde{r}_{1 l}^{R^{+}}-\Gamma_{1}^{R} \Gamma_{1}^{R^{+}}-1\right]\left(h_{0}\left(\varepsilon-e V_{1}\right)-h_{0}(\varepsilon)\right)\right\},
\end{aligned}
$$

while for the cross-current we obtain

$$
\begin{aligned}
& I_{12}\left(V_{2}\right)=-\frac{G_{0}}{4 e} \int d \varepsilon\left(1-\tanh ^{2} i L \Omega / v_{F}\right) \times \\
& \times \operatorname{Sp}\left[K _ { 2 1 } ^ { - 1 } \left\{S_{2^{\prime} 2} S_{2^{\prime} 2}^{+}\left(h_{0}\left(\varepsilon+e V_{2}\right)-h_{0}(\varepsilon)\right)+\right.\right. \\
&\left.+|a|^{2} S_{2^{\prime} 2^{\prime}} \sigma_{2} \underline{S}_{22^{\prime}}^{+} \underline{S}_{22^{\prime}} \sigma_{2} S_{2^{\prime} 2^{\prime}}^{+}\left(h_{0}\left(\varepsilon-e V_{2}\right)-h_{0}(\varepsilon)\right)\right\} K_{21}^{+-1} \times \\
&\left.\times\left(S_{11^{\prime}}^{+} S_{11^{\prime}}-\left|a^{2}\right| S_{1^{\prime} 1^{\prime}}^{+} \sigma_{2} \underline{S}_{1^{\prime} 1} \underline{S}_{1^{\prime} 1}^{+} \sigma_{2} S_{1^{\prime} 1^{\prime}}\right)\right] . \quad
\end{aligned}
$$

Eqs. (62)-64) fully determine the current across the first interface at arbitrary voltages, temperature and spindependent interface transmissions.
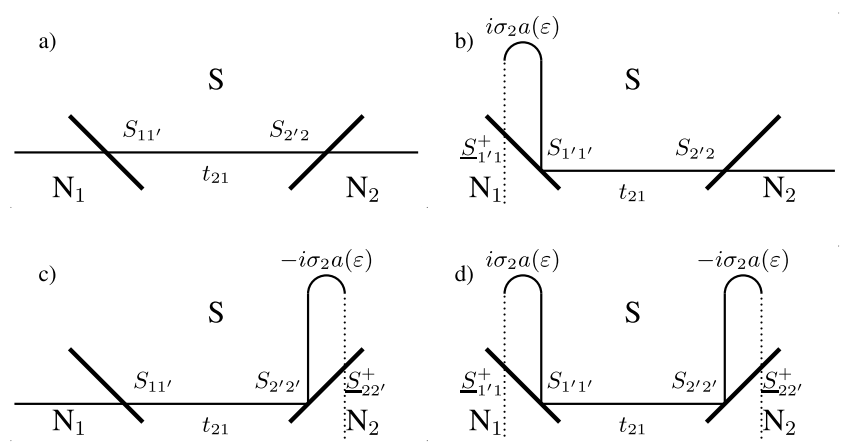

FIG. 5: Diagrams representing four different contributions to the cross-current $I_{12}$ (64). Solid (dotted) lines correspond to propagating electron-like (hole-like) excitations and $t_{21}=$ $K_{21}^{-1} / \cosh \left(i L \Omega / v_{F}\right)$.

In right hand side of Eq. (64) we can distinguish four contributions with different products of $S$-matrices. Each of these terms corresponds to a certain sequence of elementary events, such as transmission, reflection, Andreev reflection and propagation between interfaces. Diagrammatic representation of these four terms is offered in Fig. 5. The amplitude of each of the processes is given by the product of the amplitudes of the corresponding elementary events. For instance, the amplitude of the process in Fig. $5 \mathrm{c}$ is $f=-i S_{11^{\prime}} t_{21} S_{2^{\prime} 2^{\prime}} a \sigma_{2} \underline{S}_{22^{\prime}}^{+}$. In Eq.(64) this process is identified by the term $\operatorname{Sp}\left(f f^{+}\right)$with the hole distribution function as a prefactor. It is straightforward to observe that the processes of Fig. $5 \mathrm{a}, 5 \mathrm{~b}$ and $5 \mathrm{~d}$ correspond to the other three terms in (64). We also note that the processes of Fig. 5a and $5 \mathrm{~d}$ describe direct electron (hole) transport, while the processes of Fig. 5b and $5 \mathrm{c}$ correspond to the contribution of CAR.

Assuming that both interfaces possess inversion symmetry as well as reflection symmetry in the plane normal to the corresponding interface we can choose $\mathcal{S}$-matrices in the following form

$$
\begin{aligned}
& S_{11}=S_{1^{\prime} 1^{\prime}}=\underline{S}_{11}^{T}=\underline{S}_{1^{\prime} 1^{\prime}}^{T}= \\
& =U(\varphi)\left(\begin{array}{cc}
\sqrt{R_{1 \uparrow}} e^{i \theta_{1} / 2} & 0 \\
0 & \sqrt{R_{1 \downarrow}} e^{-i \theta_{1} / 2}
\end{array}\right) U^{+}(\varphi), \\
& S_{11^{\prime}}=S_{1^{\prime} 1}=\underline{S}_{11^{\prime}}^{T}=\underline{S}_{1^{\prime} 1}^{T}= \\
& =U(\varphi) i\left(\begin{array}{cc}
\sqrt{D_{1 \uparrow}} e^{i \theta_{1} / 2} & 0 \\
0 & \sqrt{D_{1 \downarrow}} e^{-i \theta_{1} / 2}
\end{array}\right) U^{+}(\varphi),
\end{aligned}
$$

and

$$
\begin{aligned}
S_{22}=S_{2^{\prime} 2^{\prime}}= & \underline{S}_{22}=\underline{S}_{2^{\prime} 2^{\prime}}= \\
& =\left(\begin{array}{cc}
\sqrt{R_{2 \uparrow}} e^{i \theta_{2} / 2} & 0 \\
0 & \sqrt{R_{2 \downarrow}} e^{-i \theta_{2} / 2}
\end{array}\right), \\
S_{22^{\prime}}=S_{2^{\prime} 2}= & \underline{S}_{22^{\prime}}=\underline{S}_{2^{\prime} 2}= \\
& =i\left(\begin{array}{cc}
\sqrt{D_{2 \uparrow}} e^{i \theta_{2} / 2} & 0 \\
0 & \sqrt{D_{2 \downarrow}} e^{-i \theta_{2} / 2}
\end{array}\right) .
\end{aligned}
$$


Here $R_{1(2) \uparrow(\downarrow)}=1-D_{1(2) \uparrow(\downarrow)}$ are the spin dependent reflection coefficients of both NS interfaces, $\theta_{1,2}$ are spinmixing angles and $U(\varphi)$ is the rotation matrix in the spin space which depends on the angle $\varphi$ between polarizations of the two interfaces,

$$
U(\varphi)=\exp \left(-i \varphi \sigma_{1} / 2\right)=\left(\begin{array}{cc}
\cos (\varphi / 2) & -i \sin (\varphi / 2) \\
-i \sin (\varphi / 2) & \cos (\varphi / 2)
\end{array}\right)
$$

Before turning to a detailed calculation of the electric current let us briefly address the issue of the spin current conservation. It is worth pointing out that in general the spin current needs not to be conserved in heterostructures with spin active interfaces, see, e.g., Ref. 33 and further references therein. Only in certain specific situations such conservation can take place. For instance, one can easily check that a single barrier with $S$-matrix (67)-(68) preserves the spin current conservation ${ }^{34}$. Making use of the general expressions for the Green-Keldysh functions we have verified that in our two barrier structure with interface $S$-matrices (65)-(68) the spin current is in general not conserved even in the normal state.
For instance, spin accumulation on the first barrier is controlled by the combination $\left(D_{2 \uparrow}-D_{2 \downarrow}\right) \sin \varphi$ which vanishes only for collinear barrier polarizations or spinisotropic second interface. We see no particular reasons to expect conservation of the spin current in general NSN structures with non-collinear interface polarizations. However, this general case requires a more detailed analysis which goes beyond the frames of the present paper and will be published elsewhere.

Now we turn to the analysis of the electric current. Substituting the above expressions for the $S$-matrices into Eqs. (63) and (64) we arrive at the final results for both $I_{11}\left(V_{1}\right)$ and $I_{12}\left(V_{2}\right)$ which will be specified further below.

\section{B. Cross-current}

First let us consider the cross-current $I_{12}\left(V_{2}\right)$. From the above analysis we obtain

$$
\begin{aligned}
I_{12}\left(V_{2}\right)=-\frac{G_{0}}{4 e} \int & d \varepsilon\left[\tanh \frac{\varepsilon+e V_{2}}{2 T}-\tanh \frac{\varepsilon}{2 T}\right] \frac{1-\tanh ^{2} i L \Omega / v_{F}}{W\left(z_{1}, z_{2}, \varepsilon, \varphi\right)} \times \\
\times & \left\{\left[D_{1 \downarrow} D_{2 \downarrow}-|a|^{2} D_{1 \uparrow} D_{2 \downarrow}\left(R_{1 \downarrow}+R_{2 \uparrow}\right)+|a|^{4} D_{1 \downarrow} R_{1 \uparrow} D_{2 \downarrow} R_{2 \uparrow}\right]\left|K\left(z_{1}, z_{2}, \varepsilon\right)\right|^{2} \cos ^{2}(\varphi / 2)+\right. \\
& +\left[D_{1 \uparrow} D_{2 \uparrow}-|a|^{2} D_{1 \downarrow} D_{2 \uparrow}\left(R_{1 \uparrow}+R_{2 \downarrow}\right)+|a|^{4} D_{1 \uparrow} R_{1 \downarrow} D_{2 \uparrow} R_{2 \downarrow}\right]\left|K\left(z_{1}^{*}, z_{2}^{*}, \varepsilon\right)\right|^{2} \cos ^{2}(\varphi / 2)+ \\
& +\left[D_{1 \uparrow} D_{2 \downarrow}-|a|^{2} D_{1 \downarrow} D_{2 \downarrow}\left(R_{1 \uparrow}+R_{2 \uparrow}\right)+|a|^{4} D_{1 \uparrow} R_{1 \downarrow} D_{2 \downarrow} R_{2 \uparrow}\right]\left|K\left(z_{1}^{*}, z_{2}, \varepsilon\right)\right|^{2} \sin ^{2}(\varphi / 2)+ \\
& \left.+\left[D_{1 \downarrow} D_{2 \uparrow}-|a|^{2} D_{1 \uparrow} D_{2 \uparrow}\left(R_{1 \downarrow}+R_{2 \downarrow}\right)+|a|^{4} D_{1 \downarrow} R_{1 \uparrow} D_{2 \uparrow} R_{2 \downarrow}\right]\left|K\left(z_{1}, z_{2}^{*}, \varepsilon\right)\right|^{2} \sin ^{2}(\varphi / 2)\right\},
\end{aligned}
$$

where we define

$$
\begin{gathered}
K\left(z_{1}, z_{2}, \varepsilon\right)=\left(1-a^{2} z_{1} z_{2}\right)-\left[\varepsilon\left(1+a^{2} z_{1} z_{2}\right)+\Delta a\left(z_{1}+z_{2}\right)\right] Q \\
W\left(z_{1}, z_{2}, \varepsilon, \varphi\right)=\left|K\left(z_{1}, z_{2}, \varepsilon\right) K\left(z_{1}^{*}, z_{2}^{*}, \varepsilon\right) \cos ^{2}(\varphi / 2)+K\left(z_{1}^{*}, z_{2}, \varepsilon\right) K\left(z_{1}, z_{2}^{*}, \varepsilon\right) \sin ^{2}(\varphi / 2)\right|^{2}
\end{gathered}
$$

and $z_{i}=\sqrt{R_{i \uparrow} R_{i \downarrow}} \exp \left(i \theta_{i}\right)(i=1,2)$.

Eq. (70) represents our central result. It fully determines the non-local spin-dependent current in our threeterminal ballistic NSN structure at arbitrary voltages, temperature, interface transmissions and polarizations.

Let us introduce the non-local differential conductance

$$
G_{12}\left(V_{2}\right)=-\frac{\partial I_{1}}{\partial V_{2}}=-\frac{\partial I_{12}\left(V_{2}\right)}{\partial V_{2}} .
$$

Before specifying this quantity further it is important to observe that in general the conductance $G_{12}\left(V_{2}\right)$ is not an even function of the applied voltage $V_{2}$. This asymmetry arises due to formation of Andreev bound states in the vicinity of a spin-active interface ${ }^{35.36}$. It disappears provided the spin mixing angles $\theta_{1}$ and $\theta_{2}$ remain equal to 0 or $\pi$.

In the normal state we have $I_{12}\left(V_{2}\right)=-G_{N_{12}} V_{2}$, where

$$
\begin{aligned}
G_{N_{12}}=\frac{G_{0}}{2}[( & \left.D_{1 \downarrow} D_{2 \downarrow}+D_{1 \uparrow} D_{2 \uparrow}\right) \cos ^{2}(\varphi / 2)+ \\
& \left.+\left(D_{1 \uparrow} D_{2 \downarrow}+D_{1 \downarrow} D_{2 \uparrow}\right) \sin ^{2}(\varphi / 2)\right] .
\end{aligned}
$$

Turning to the superconducting state, let us consider the limit of low temperatures and voltage $T, V_{2} \ll \Delta$. In this limit only subgap quasiparticles contribute to the cross-current and the differential conductance becomes voltage-independent, i.e. $I_{12}=-G_{12} V_{2}$, where 


$$
\begin{aligned}
& G_{12}=G_{0}\left(1-\tanh ^{2} L \Delta\right.\left./ v_{F}\right)\left\{\frac{D_{1 \uparrow} D_{1 \downarrow} D_{2 \uparrow} D_{2 \downarrow}}{\left|K\left(z_{1}, z_{2}, 0\right)\right|^{2} \cos ^{2}(\varphi / 2)+\left|K\left(z_{1}, z_{2}^{*}, 0\right)\right|^{2} \sin ^{2}(\varphi / 2)}+\right. \\
&\left.+\left(D_{1 \uparrow}-D_{1 \downarrow}\right)\left(D_{2 \uparrow}-D_{2 \downarrow}\right) \frac{\left|K\left(z_{1}, z_{2}, 0\right)\right|^{2} \cos ^{2}(\varphi / 2)-\left|K\left(z_{1}, z_{2}^{*}, 0\right)\right|^{2} \sin ^{2}(\varphi / 2)}{\left(\left|K\left(z_{1}, z_{2}, 0\right)\right|^{2} \cos ^{2}(\varphi / 2)+\left|K\left(z_{1}, z_{2}^{*}, 0\right)\right|^{2} \sin ^{2}(\varphi / 2)\right)^{2}}\right\} .
\end{aligned}
$$

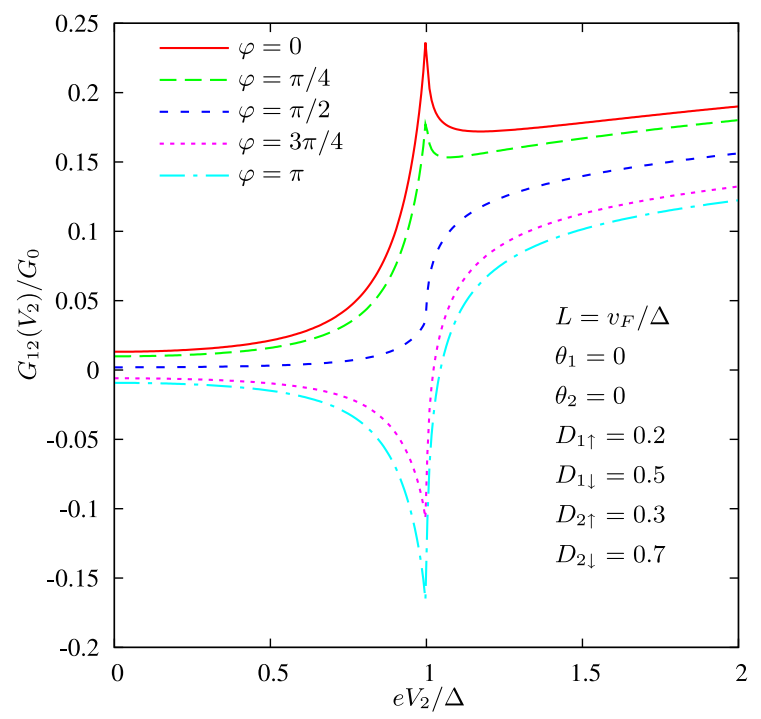

FIG. 6: Zero temperature differential non-local conductance as a function of voltage at zero spin-mixing angles $\theta_{1,2}=0$.

In the case of spin-isotropic interfaces Eqs. (75) and (70) reduce to our previous results 12 .

Provided at least one of the interfaces is spin-isotropic, the conductance (75) is proportional to the product of all four transmissions $D_{1 \uparrow} D_{1 \downarrow} D_{2 \uparrow} D_{2 \downarrow}$, i.e. it differs from zero only due to processes involving scattering with both spin projections at both NS interfaces. As in the case of spin-isotropic interfaces $\frac{12}{2}$ the value $G_{12}$ (75) gets strongly suppressed with increasing $L$, and at sufficiently high interface transmissions this dependence is in general non-exponential in $L$. In the spin-degenerate case for a given $L$ the non-local conductance reaches its maximum for reflectionless barriers $D_{1,2}=1$. In this case we arrive at a simple formula

$$
G_{12}=G_{0}\left(1-\tanh ^{2} L \Delta / v_{F}\right)
$$

We observe that for small $L \ll v_{F} / \Delta$ the conductance $G_{12}$ identically coincides with its normal state value $G_{N_{12}} \equiv G_{0}$ at any temperature and voltage $\frac{12}{2}$. This result implies that CAR vanishes for fully open barriers. Actually this conclusion is general and applies not only for small but for any value of $L$, i.e. the result (76) is determined solely by the process of direct electron transfer between N-terminals for all $L$.
At the first sight, this result might appear counterintuitive since the behavior of ordinary (local) AR is just the opposite: It reaches its maximum at full barrier transmissions. The physics behind vanishing of CAR for perfectly trasparent NS interfaces is simple. One observes (cf. Fig. 1) that CAR inevitably implies the flow of Cooper pairs out of the contact area into the superconducting terminal. This flow is described by electron trajectories which end deep in the superconductor. On the other hand, it is obvious that CAR requires "mixing" of these trajectories with those going straight between two normal terminals. Provided there exists no normal electron reflection at both NS interfaces such mixing does not occur, CAR vanishes and the only remaining contribution to the non-local conductance is one from direct electron transfer between N-terminals.

This situation is illustrated by the diagrams in Fig. 5. It is obvious that in the case of non-reflecting NS interfaces only the process of Fig. 5a survives, whereas all other processes (Fig. 5b, 5c and 5d) vanish for reflectionless barriers with $R_{1(2) \uparrow(\downarrow)}=0$. The situation changes provided at least one of the transmissions is smaller than one. In this case scattering at $\mathrm{SN}$ interfaces mixes up trajectories connecting $\mathrm{N}_{1}$ and $\mathrm{N}_{2}$ terminals with ones going deep into and coming from the superconductor. As a result, all four processes depicted in Fig. 5 contribute to the cross-current and CAR contribution to $G_{12}$ does not vanish.

Let us also note that the statement about the absence of CAR for highly transparent interfaces was independently made in a recent paper ${ }^{37}$. Although no derivation supporting this statement was presented, this statement $\underline{37}$ appears to be based on the BTK-like description of strictly 1d NSN structures. According to our general analysis, no processes presented in Figs. 5b, 5c and $5 \mathrm{~d}$ would be possible in that case and, hence, CAR should be prohibited in 1d NSN systems for any transmission. This observation, however, can be easily overlooked if one only deals with the solutions of the Bogolyubovde Gennes equations (equivalent to finding retarded and advanced Green functions) and does not directly evaluate the electron distribution function (or the Keldysh function) in the S-terminal. 


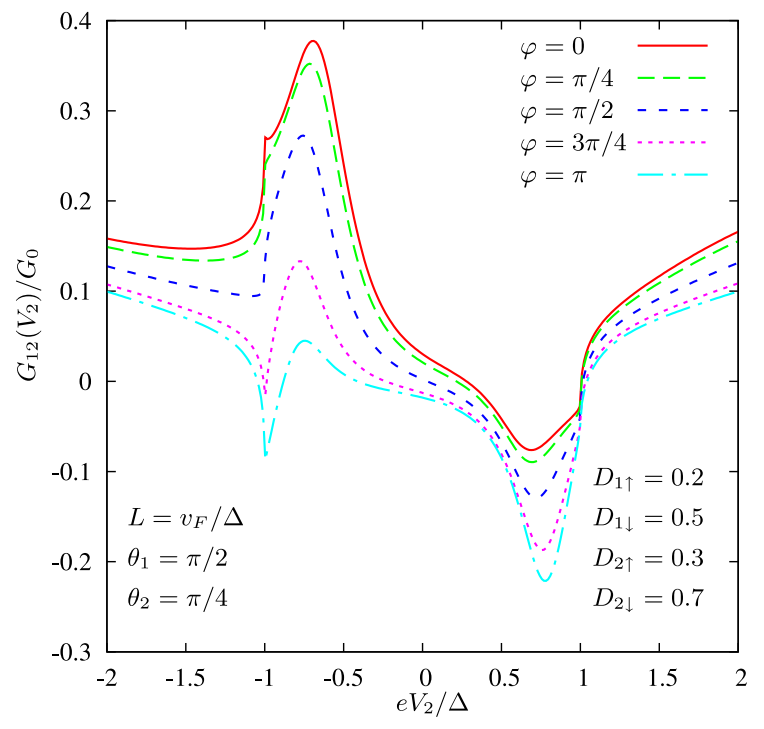

FIG. 7: The same as in Fig. 6 for $\theta_{1}=\pi / 2, \theta_{2}=\pi / 4$.

In the limit $\left|e V_{2}\right|, T \ll \Delta$ and at zero spin-mixing angles $\theta_{1,2}=0$ from Eq. (75) we obtain

$$
\begin{array}{r}
G_{12}=G_{0} \frac{1-\tanh ^{2} L \Delta / v_{F}}{\left|K\left(z_{1}, z_{2}, 0\right)\right|^{2}}\left\{D_{1 \uparrow} D_{1 \downarrow} D_{2 \uparrow} D_{2 \downarrow}+\right. \\
\left.+\left(D_{1 \uparrow}-D_{1 \downarrow}\right)\left(D_{2 \uparrow}-D_{2 \downarrow}\right) \cos \varphi\right\} .
\end{array}
$$

In the lowest (first order) order in the transmissions of both interfaces and for collinear interface polarizations Eq. (77) reduces to the result by Falci et al. $\stackrel{8}{=}$ provided we identify the tunneling density of states $N_{0} D_{1 \uparrow}$, $N_{0} D_{1 \downarrow}, N_{0} D_{2 \uparrow}$, and $N_{0} D_{2 \downarrow}$ with the corresponding spinresolved densities of states in the ferromagnetic electrodes. For zero spin-mixing angles and low voltages the $L$-dependence of the nonlocal conductance $G_{12}$ reduces to the exponential form $G_{12} \propto \exp \left(-2 L \Delta / v_{F}\right)$ either in the limit of small transmissions or large $L \gg v_{F} / \Delta$.

At arbitrary voltages and temperatures the crosscurrent has a simple $\varphi$ dependence in the limit of zero spin mixing angles $\left(\theta_{1,2}=0\right)$

$$
\begin{aligned}
I_{12}\left(\varphi, V_{2}\right)=I_{12}(\varphi= & \left.0, V_{2}\right) \cos ^{2}(\varphi / 2)+ \\
& +I_{12}\left(\varphi=\pi, V_{2}\right) \sin ^{2}(\varphi / 2),
\end{aligned}
$$

i.e. in this limit at any $\varphi$ the nonlocal current is equal to a proper superposition of the two contributions corresponding to parallel $(\varphi=0)$ and antiparallel $(\varphi=\pi)$ interface polarizations. Some typical curves for the differential non-local conductance are presented in Fig. 6 at sufficiently high interface transmissions and zero spin mixing angles $\theta_{1,2}=0$.

Let us now turn to the limit of highly polarized interfaces which is accounted for by taking the limit of vanishing spin-up (or spin-down) transmission of each interface. In this limit our model describes an HSH structure, where $\mathrm{H}$ stands for fully spin-polarized half-metallic electrodes.
In this case we obtain $\left(D_{1 \uparrow}=D_{1}, D_{1 \downarrow}=0, D_{2 \uparrow}=D_{2}\right.$, and $D_{2 \downarrow}=0$ )

$$
\begin{aligned}
& I_{12}\left(V_{2}\right)=- \frac{G_{0}}{4 e} \int d \varepsilon\left[h_{0}\left(\varepsilon+e V_{2}\right)-h_{0}(\varepsilon)\right] \times \\
& \times \frac{1-\tanh ^{2} i L \Omega / v_{F}}{W\left(z_{1}, z_{2}, \varepsilon, \varphi\right)} D_{1} D_{2} \times \\
& \times\left\{\left[1+|a|^{4}\right]\left|K\left(z_{1}^{*}, z_{2}^{*}, \varepsilon\right)\right|^{2} \cos ^{2}(\varphi / 2)-\right. \\
&\left.\quad-2|a|^{2}\left|K\left(z_{1}, z_{2}^{*}, \varepsilon\right)\right|^{2} \sin ^{2}(\varphi / 2)\right\} .
\end{aligned}
$$

We observe that the nonlocal conductance has opposite signs for parallel $(\varphi=0)$ and antiparallel $(\varphi=\pi)$ interface polarizations. We also emphasize that, as it is also clear from Eq. (77), the cross-conductance $G_{12}$ of HSH structures - in contrast to that for NSN structures - does not vanish already in the lowest order in barrier transmissions $D_{1 \uparrow} D_{2 \uparrow}$.

In general the non-local conductance is very sensitive to particular values of the spin-mixing angles $\theta_{1}$ and $\theta_{2}$, as illustrated, e.g., in Fig. 7. Comparing the voltage dependencies of the nonlocal conductance evaluated for the same transmissions and presented in Figs. 6 and 7 , we observe that they can differ drastically at zero and non-zero values of $\theta_{1,2}$.

At low voltages and temperatures and at zero spin mixing angles the non-local conductance of HSH structures is determined by Eq. (77) with $D_{1 \downarrow}=D_{2 \downarrow}=0$. For fully open barriers (for "spin-up" electrons) $D_{1 \uparrow}=D_{2 \uparrow}=1$ we obtain

$$
G_{12}=G_{0}\left(1-\tanh ^{2} L \Delta / v_{F}\right) \cos \varphi .
$$

Interestingly, for $\varphi=0$ this expression exactly coincides with that for fully open NSN structures, Eq. (76). At the same time for small $L$ the result (80) turns out to be 2 times bigger that the analogous expression in the normal case, i.e. for fully open HNH structures, cf. Eq. (74). This result can easily be interpreted in terms of diagrams in Fig. 5. We observe that - exactly as for the spin degenerate case - CAR diagrams of Fig. 5b,c vanish for reflectionless barriers, whereas diagrams of Fig. $5 \mathrm{a}, \mathrm{d}$ describing direct electron transfer survive and both contribute to $G_{12}$. Thus, CAR vanishes identically also for fully open HSH structures. The factor of 2 difference with the normal case is due to the fact that the diagram of Fig. 5d vanishes in the normal limit.

Finally, let us compare our general results, Eq. (70) and below, with the the analogous results 10 . In order to account for higher order tunneling events in the case of symmetric interfaces the authors $\frac{10}{0}$ employed effective "dressing" of both EC and CAR contributions by all higher order local Andreev processes at both interfaces. At the same time, all higher order non-local processes were ignored in Ref. 10. Our analysis - which includes all processes to all orders - demonstrates that this approximation $\frac{10}{}$ may be appropriate only in the limit 
$L \gg v_{F} / \Delta$ in which case non-local effects are exponentially suppressed. Otherwise higher order non-local processes remain important and need to be fully accounted for.

Comparing our exact results and those of Ref. 10 (with omitted higher order non-local processes) we observe that the disagreement between them grows with increasing transmissions and becomes maximal at full transmissions and small values of $L$. The most significant differences are: (i) the dependence of $G_{12}$ on $L$ is non-exponential whereas in Ref. 10 it remains exponential at all $L$, (ii) at small $L$ and high transmissions the linear conductance $G_{12}$ evaluated in Ref. 10 (see e.g. Eqs. (33), (34) of that work) turns out to be several times smaller than our results in both cases of NSN and HSH structures and (iii) taking into account only local Andreev reflection events is not sufficient to correctly account for vanishing CAR in the limit of fully open interfaces.

\section{Correction to BTK}

Using the above formalism one can easily generalize the BTK result to the case of spin-polarized interfaces 31 .
For the first interface we have

$$
\begin{aligned}
& I_{1}^{B T K}\left(V_{1}\right)=\frac{\mathcal{N}_{1}}{R_{q} e} \int d \varepsilon\left[h_{0}\left(\varepsilon+e V_{1}\right)-h_{0}(\varepsilon)\right]\left(1+|a|^{2}\right) \times \\
& \times\left\langle\frac{\left|v_{x_{1}}\right|}{v_{F}}\left(D_{1 \uparrow} \frac{1-R_{1 \downarrow}|a|^{2}}{\left|1-z_{1} a^{2}\right|^{2}}+D_{1 \downarrow} \frac{1-R_{1 \uparrow}|a|^{2}}{\left|1-z_{1}^{*} a^{2}\right|^{2}}\right)\right\rangle \cdot \quad
\end{aligned}
$$

Here transmission and reflection coefficients as well as the spin mixing angle depend on the direction of the Fermi momentum. In the spin-degenerate case the above expression reduces to the standard BTK result ${ }^{2}$.

Evaluating the nonlocal correction to the BTK current due to the presence of the second interface we arrive at a somewhat lengthy general expression

$$
\begin{aligned}
I_{11}\left(V_{1}\right)=\frac{G_{0}}{2 e} \int d \varepsilon\left(h_{0}\left(\varepsilon+e V_{1}\right)-h_{0}(\varepsilon)\right) \frac{1}{W\left(z_{1}, z_{2}, \varepsilon, \varphi\right)}\left\{2 W\left(z_{1}, z_{2}, \varepsilon, \varphi\right)-\right. \\
-R_{1 \uparrow}\left|\cos ^{2}(\varphi / 2) K\left(z_{1} / R_{1 \uparrow}, z_{2}, \varepsilon\right) K\left(z_{1}^{*}, z_{2}^{*}, \varepsilon\right)+\sin ^{2}(\varphi / 2) K\left(z_{1} / R_{1 \uparrow}, z_{2}^{*}, \varepsilon\right) K\left(z_{1}^{*}, z_{2}, \varepsilon\right)\right|^{2}- \\
\left.-R_{1 \downarrow}\left|\cos ^{2}(\varphi / 2) K\left(z_{1}^{*} / R_{1 \downarrow}, z_{2}^{*}, \varepsilon\right) K\left(z_{1}, z_{2}, \varepsilon\right)+\sin ^{2}(\varphi / 2) K\left(z_{1}^{*} / R_{1 \downarrow}, z_{2}, \varepsilon\right) K\left(z_{1}, z_{2}^{*}, \varepsilon\right)\right|^{2}\right\}+ \\
+\frac{G_{0}}{4 e} \int d \varepsilon\left(h_{0}\left(\varepsilon+e V_{1}\right)-h_{0}(\varepsilon)\right) \frac{D_{1 \uparrow} D_{1 \downarrow}}{W\left(z_{1}, z_{2}, \varepsilon, \varphi\right)}\{ \\
|a|^{2}\left|\cos ^{2}(\varphi / 2) K\left(0, z_{2}, \varepsilon\right) K\left(z_{1}^{*}, z_{2}^{*}, \varepsilon\right)+\sin ^{2}(\varphi / 2) K\left(0, z_{2}^{*}, \varepsilon\right) K\left(z_{1}^{*}, z_{2}, \varepsilon\right)\right|^{2}+ \\
+|a|^{2}\left|\cos ^{2}(\varphi / 2) K\left(0, z_{2}^{*}, \varepsilon\right) K\left(z_{1}, z_{2}, \varepsilon\right)+\sin ^{2}(\varphi / 2) K\left(0, z_{2}, \varepsilon\right) K\left(z_{1}, z_{2}^{*}, \varepsilon\right)\right|^{2}+ \\
+\frac{1}{|a|^{2}}\left|\cos ^{2}(\varphi / 2) K^{\prime}\left(z_{2}^{*}, \varepsilon\right) K\left(z_{1}, z_{2}, \varepsilon\right)+\sin ^{2}(\varphi / 2) K^{\prime}\left(z_{2}, \varepsilon\right) K\left(z_{1}, z_{2}^{*}, \varepsilon\right)\right|^{2}+ \\
\left.+\frac{1}{|a|^{2}}\left|\cos ^{2}(\varphi / 2) K^{\prime}\left(z_{2}, \varepsilon\right) K\left(z_{1}^{*}, z_{2}^{*}, \varepsilon\right)+\sin ^{2}(\varphi / 2) K^{\prime}\left(z_{2}^{*}, \varepsilon\right) K\left(z_{1}^{*}, z_{2}, \varepsilon\right)\right|^{2}\right\}+ \\
+\frac{G_{0}}{e} R_{2 \uparrow} R_{2 \downarrow} \sin ^{2}\left(\theta_{2} / 2\right) \sin ^{2}(\varphi / 2) \cos ^{2}(\varphi / 2) \int d \varepsilon\left(h_{0}\left(\varepsilon+e V_{1}\right)-h_{0}(\varepsilon)\right) \frac{\left(1-\tanh ^{2} i L \Omega / v_{F}\right)^{2}}{W\left(z_{1}, z_{2}, \varepsilon, \varphi\right)} \times \\
\times\left[|a|^{2}\left(D_{1 \uparrow}^{2}+D_{1 \downarrow}^{2}\right)-2|a|^{4} D_{1 \uparrow} D_{1 \downarrow}\left(R_{1 \uparrow}+R_{1 \downarrow}\right)+|a|^{6}\left(D_{1 \uparrow}^{2} R_{1 \downarrow}^{2}+D_{1 \downarrow}^{2} R_{1 \uparrow}^{2}\right)\right]
\end{aligned}
$$

where $K^{\prime}\left(z_{2}, \varepsilon\right)=\partial K\left(z_{1}, z_{2}, \varepsilon\right) / \partial z_{1}$. This expression gets significantly simplified in the limit of zero spin- mixing angles $\theta_{1,2}=0$ in which case we obtain

$$
\begin{aligned}
& I_{11}\left(V_{1}\right)=\frac{G_{0}}{2 e} \int d \varepsilon\left(h_{0}\left(\varepsilon+e V_{1}\right)-h_{0}(\varepsilon)\right) \\
& \left\{2-R_{1 \uparrow} \frac{\left|K\left(z_{1} / R_{1 \uparrow}, z_{2}, \varepsilon\right)\right|^{2}}{\left|K\left(z_{1}, z_{2}, \varepsilon\right)\right|^{2}}-R_{1 \downarrow} \frac{\left|K\left(z_{1} / R_{1 \downarrow}, z_{2}, \varepsilon\right)\right|^{2}}{\left|K\left(z_{1}, z_{2}, \varepsilon\right)\right|^{2}}+\right. \\
& \left.+D_{1 \uparrow} D_{1 \downarrow} \frac{|a(\varepsilon)|^{2}\left|K\left(0, z_{2}, \varepsilon\right)\right|^{2}+\left|K^{\prime}\left(z_{2}, \varepsilon\right)\right|^{2} /|a(\varepsilon)|^{2}}{\left|K\left(z_{1}, z_{2}, \varepsilon\right)\right|^{2}}\right\} .
\end{aligned}
$$


In contrast to the expression for the cross-current $I_{12}$ (cf. Eq. (78)), in the limit of zero spin-mixing angles the correction $I_{11}$ to the BTK current does not depend on the angle $\varphi$ between the interface polarizations. In particular, at $\left|e V_{1}\right|, T \ll \Delta$ we have $I_{11}=G_{11} V_{1}$ where

$$
\begin{gathered}
G_{11}=G_{0}\left(D_{1 \uparrow}+D_{1 \downarrow}\right) \frac{\left(1-z_{2}^{2}\right)\left(1-\tanh ^{2} L \Delta / v_{F}\right)}{\left[1+z_{1} z_{2}+\left(z_{1}+z_{2}\right) \tanh L \Delta / v_{F}\right]^{2}}+ \\
+G_{0} D_{1 \uparrow} D_{1 \downarrow} \frac{\left(1+z_{2} \tanh L \Delta / v_{F}\right)^{2}+3\left(z_{2}+\tanh L \Delta / v_{F}\right)^{2}}{\left[1+z_{1} z_{2}+\left(z_{1}+z_{2}\right) \tanh L \Delta / v_{F}\right]^{2}} .
\end{gathered}
$$

In the tunneling limit $D_{1 \uparrow}, D_{1 \downarrow}, D_{2 \uparrow}, D_{2 \downarrow} \ll 1$ we reproduce the result of Ref. 8

$$
G_{11}=\frac{G_{0}}{4}\left(D_{1 \uparrow}+D_{1 \downarrow}\right)\left(D_{2 \uparrow}+D_{2 \downarrow}\right) \exp \left(-2 L \Delta / v_{F}\right),
$$

which turns out to hold at any value $\varphi$.

As compared to the BTK conductance the CAR correction (82) contains an extra small factor $\mathcal{A}_{2} / L^{2}$ and, hence, in many cases remains small and can be neglected. On the other hand, since CAR involves tunneling of one electron through each interface, for strongly asymmetric structures with $D_{1 \uparrow}, D_{1 \downarrow} \ll 1$ and $D_{2 \uparrow}, D_{2 \downarrow} \sim 1$ it can actually strongly exceed the BTK conductance. Indeed, for $D_{1 \uparrow \downarrow} \ll 1, R_{2 \uparrow} R_{2 \downarrow} \ll 1$ and provided the spin mixing angle $\theta_{1}$ is not very close to $\pi$ from Eq. (82) we get

$$
G_{11}=\frac{G_{0}\left(D_{1 \uparrow}+D_{1 \downarrow}\right)}{\cosh \left(2 L \Delta / v_{F}\right)+\cos \theta_{1} \sinh \left(2 L \Delta / v_{F}\right)},
$$

i.e. for

$$
\frac{D_{1 \uparrow} D_{1 \downarrow}}{\left(D_{1 \uparrow}+D_{1 \downarrow}\right)}<\frac{\mathcal{A}_{2}}{L^{2}} \exp \left(-2 L \Delta / v_{F}\right)
$$

the contribution (86) may well exceed the BTK term $G_{1}^{B T K} \propto D_{1 \uparrow} D_{1 \downarrow}$. The existence of such a non-trivial regime further emphasizes the importance of the mechanism of non-local Andreev reflection in multi-terminal hybrid NSN structures.

\section{CONCLUSIONS}

In this paper we developed a non-perturbative theory of non-local electron transport in ballistic NSN three-terminal structures with spin-active interfaces. Our theory is based on the quasiclassical formalism of energy-integrated Green-Eilenberger functions supplemented by appropriate boundary conditions describing spin-dependent scattering at NS interfaces. Our approach applies at arbitrary interface transmissions and allows to fully describe non-trivial interplay between + spin-sensitive normal scattering, local and non-local Andreev reflection at SN interfaces. Our main results are the general expressions for the non-local cross-current $I_{12}$, Eq. (70), and for the non-local correction $I_{11}$ to the BTK current, Eq. (82). These expressions provide complete description of the conductance matrix of our three-terminal NSN device at arbitrary voltages, temperature, spin-dependent transmissions of NS interfaces and their polarizations.

Our analysis allows to predict and analyze a rich variety of interesting properties of such structures. One of our predictions is that in the case of ballistic electrodes no crossed Andreev reflection can occur in both NSN and HSH structures with fully open interfaces. Beyond the tunneling limit the dependence of the non-local conductance on the size of the S-electrode $L$ is in general non-exponential and reduces to $G_{12} \propto \exp \left(-2 L \Delta / v_{F}\right)$ only in the limit of large $L$. For hybrid structures halfmetal-superconductor-half-metal we predict that the low energy non-local conductance does not vanish already in the lowest order in barrier transmissions $G_{12} \propto D_{1 \uparrow} D_{2 \uparrow}$. These and other our predictions can be directly tested in future experiments on NSN hybrid structures, including systems with ferromagnetic and half-metallic electrodes.

\section{Acknowledgments}

We are indebted to D. Beckmann for pointing out numerical mistakes in initial versions of Fig. 6 and 7. This work is part of the EU Framework Programme NMP4CT-2003-505457 ULTRA-1D "Experimental and theoretical investigation of electron transport in ultra-narrow 1-dimensional nanostructures".
1 A.F. Andreev, Zh. Eksp. Teor. Fiz. 46, 1823 (1964) [Sov. Phys. JETP 19, 1228 (1964)].

2 G.E. Blonder, M. Tinkham, and T.M. Klapwijk, Phys. Rev. B 25, 4515 (1982).

3 J.M. Byers and M.E. Flatte, Phys. Rev. Lett. 74, 306 (1995).

${ }^{4}$ G. Deutscher and D. Feinberg, Appl. Phys. Lett. 76, 487
(2000).

5 D. Beckmann, H.B. Weber, and H. v. Löhneysen, Phys. Rev. Lett. 93, 197003 (2004); D. Beckmann and H. v. Löhneysen, Applied Physics A, 89, 603 (2007).

6 S. Russo, M. Kroug, T.M. Klapwijk, and A.F. Morpurgo, Phys. Rev. Lett. 95, 027002 (2005).

7 P. Cadden-Zimansky and V. Chandrasekhar, Phys. Rev. 
Lett. 97, 237003 (2006) .

8 G. Falci, D. Feinberg, and F.W.J. Hekking, Europhys. Lett. 54, 255 (2001).

9 G. Bignon, M. Houset, F. Pistolesi, and F.W.J. Hekking, Europhys. Lett. 67, 110 (2004).

${ }^{10}$ R. Melin and D. Feinberg, Phys. Rev. B 70, 174509 (2004).

11 R. Melin, Phys. Rev. B 73, 174512 (2006).

12 M.S. Kalenkov and A.D. Zaikin, Phys. Rev. B 75, 172503 (2007); M. S. Kalenkov and A. D. Zaikin, Physica E 40, 147 (2007).

13 A.F. Volkov, A.V. Zaitsev, and T.M. Klapwijk, Physica C 210, 21 (1993).

14 F.W.J. Hekking and Yu.V. Nazarov, Phys. Rev. Lett. 71, 1625 (1993).

15 A.D. Zaikin, Phyica B 203, 255 (1994).

16 A. Brinkman and A.A. Golubov, Phys. Rev. B 74, 214512 (2006).

17 J.P. Morten, A. Brataas, and W. Belzig, Phys. Rev. B 74, 214510 (2006).

18 S. Duhot and R. Melin, Phys. Rev. B 75, 184531 (2007).

19 D.S. Golubev and A.D. Zaikin, Phys. Rev. B 76, 184510 (2007) .

20 The presence of an additional dot with vanishing superconducting order parameter ${ }^{17}$ can cause modifications of the physical picture of non-local electron transport as compared to that analyzed in other works.

21 A. Huck, F.W.J. Hekking, and B. Kramer, Europhys. Lett. 41, 201 (1998).

22 A.V. Galaktionov and A.D. Zaikin, Phys. Rev. B 73,
184522 (2006).

23 A. Levy Yeyati, F.S. Bergeret, A. Martin-Rodero, and T.M. Klapwijk, Nature Physics 3, 455 (2007).

24 T. Yamashita, S. Takahashi, and S. Maekawa, Phys. Rev. B 68, 174504 (2003).

25 F. Giazotto, F. Taddei, F. Beltram, and R. Fazio, Phys. Rev. Lett. 97, 087001 (2006).

${ }^{26}$ For a review see, e.g., W. Belzig, F.K. Wilhelm, C. Bruder, G. Schön, and A.D. Zaikin, Superlatt. Microstruct. 25, 1251 (1999).

27 A.V. Zaitsev, Sov. Phys. JETP 59, 1015 (1984).

28 A.V. Galaktionov and A.D. Zaikin, Phys. Rev. B 65, 184507 (2002).

29 M. Ozana and A. Shelankov, Phys. Rev. B 65, 014510 (2002).

30 M. Eschrig, Phys. Rev. B 61, 9061 (2000).

31 E. Zhao, T. Löfwander, and J.A. Sauls, Phys. Rev. B 70 134510 (2004).

32 A. Millis, D. Rainer, and J.A. Sauls, Phys. Rev. B 384504 (1988).

33 Y. Tserkovnyak, A. Brataas, G.E.W. Bauer, and B.I. Halperin, Rev. Mod. Phys. 77, 1375 (2005).

34 E. Zhao and J.A. Sauls, Phys. Rev. Lett. 98, 206601 (2007).

35 M. Fogelström, Phys. Rev. B 62, 11812 (2000).

36 Yu.S. Barash and I.V. Bobkova, Phys. Rev. B 65, 144502 (2002).

37 S. Duhot and R. Melin, Eur. Phys. J. B 53, 257 (2006). 